Supporting Information for

\title{
A New Twist on Pincer Ligands and Complexes
}

Liqing Ma, Robert A. Woloszynek, Weizhong Chen, Tong Ren, John D. Protasiewicz* 


\section{General Procedures and Materials}

Experiments were carried out using standard Schlenk techniques or in a glove box under nitrogen. Certified A.C.S. grade solvents (diethyl ether, $\mathrm{CCl}_{4}, \mathrm{CH}_{2} \mathrm{Cl}_{2}$, ethanol, methanol, hexanes, ethyl acetate), anhydrous acetone from Fisher were used as received. Anhydrous solvents (THF, benzene) were distilled from Na/benzophenone prior to use. The NMR spectroscopy measurements were recorded on Varian Inova 400 or $600 \mathrm{MHz}$ spectrometers. Chemical shifts given in ppm were referenced to residual solvent signals $\left({ }^{1} \mathrm{H},{ }^{13} \mathrm{C} \mathrm{NMR}\right)$ or external $85 \% \mathrm{H}_{3} \mathrm{PO}_{4}$ as reference $\left({ }^{31} \mathrm{P} \mathrm{NMR}\right)$. Elemental analyses were performed by Quantitative Technologies, Inc. NJ. Routes compound to compounds $\mathbf{i}^{[1]}$ and $\mathbf{i i -} \mathbf{v}^{[2]}$ were adapted from the literature.

\section{2,6- $\left(2-\mathrm{CH}_{3} \mathrm{C}_{6} \mathrm{H}_{4}\right)_{2}-1-\mathrm{BrC}_{6} \mathrm{H}_{3}(\mathbf{i})$}

To a solution of 2,6-dichlorophenyl-lithium [prepared by stirring 2, 6-dichlorobenzene (10.3 g, 70.1 $\mathrm{mmol})$ and ${ }^{\mathrm{n}} \mathrm{BuLi}\left(30 \mathrm{~mL}, 2.5 \mathrm{M}\right.$ in hexanes) in $150 \mathrm{~mL}$ anhydrous THF at $-78{ }^{\circ} \mathrm{C}$ for $1 \mathrm{~h}$ ], was added dropwise a solution of (2-methylphenyl) magnesium bromide [prepared from 2-bromotoluene (29.4 g, $172 \mathrm{mmol})$ and magnesium $(8.30 \mathrm{~g}, 342 \mathrm{mmol})$ in $150 \mathrm{~mL}$ anhydrous THF under room temperature for 1h]. The reaction mixture was stirred at $-78^{\circ} \mathrm{C}$ for $1 \mathrm{~h}$, then warmed to room temperature and heated to reflux under nitrogen overnight. The reaction mixture was then cooled to room temperature, and bromine (20 g, $125 \mathrm{mmol}$ ) was slowly added. The resulting solution was stirred for an additional $2 \mathrm{~h}$. Diethyl ether $(100 \mathrm{~mL})$ was added, and excess bromine was quenched with $5 \% \mathrm{NaSO}_{3}(\mathrm{aq})(3 \times 100$ $\mathrm{mL}$ ). The organic layer was separated and dried with anhydrous $\mathrm{MgSO}_{4}$. After removal of the ether under vacuum the material was washed with $n$-pentane to afford $13.2 \mathrm{~g} \mathbf{i}$ as a fine white powder (56.0 $\%)$.

(Chemical shifts of two isomers are reported without further assigning to syn- or anti-) ${ }^{1} \mathrm{H}$ NMR $\left(\mathrm{CDCl}_{3}, 400 \mathrm{MHz}\right): \delta 2.12,2.13,7.15-7.20(\mathrm{~m}, 4 \mathrm{H}), 7.22-7.31(\mathrm{~m}, 6 \mathrm{H}), 7.34-7.40(\mathrm{~m}, 1 \mathrm{H}) .{ }^{13} \mathrm{C}\left\{{ }^{1} \mathrm{H}\right\}$ 
NMR $\left(\mathrm{CDCl}_{3}, 100 \mathrm{MHz}\right): \delta$ 20.0, 20.1, 125.1, 125.4, 125.7, 125.8, 127.1, 127.2, 128.07, 128.09, 129.3, $129.5,129.8,130.0,130.1,136.2,136.3,142.0,143.6,143.7$. Elemental analysis calcd for $\mathrm{C}_{20} \mathrm{H}_{17} \mathrm{Br}$ (337.26): C, 71.23; H, 5.08. Found: C, 70.96; H, 4.91.

\section{2,6-(2-CHBr $\left.{ }_{2} \mathrm{C}_{6} \mathrm{H}_{4}\right)_{2}-1-\mathrm{BrC}_{6} \mathrm{H}_{3}$ (ii)}

To a solution of $\mathbf{i}(6.00 \mathrm{~g}, 17.8 \mathrm{mmol})$ in $200 \mathrm{~mL} \mathrm{CCl}_{4}, N$-bromosuccinimide $(7.40 \mathrm{~g}, 41.6 \mathrm{mmol})$ and benzoyl peroxide $(50 \mathrm{mg}$ ) were added. The solution was heated to reflux for $6 \mathrm{~h}$, and another portion of $N$-bromosuccinimide $(7.60 \mathrm{~g}, 42.7 \mathrm{mmol})$ and benzoyl peroxide $(50 \mathrm{mg})$ were added. After an additional $18 \mathrm{~h}$ reflux, the mixture was cooled to RT, and the succinimide was removed by filtration; the filtrate was washed with $5 \% \mathrm{NaSO}_{3}(\mathrm{aq})(3 \times 100 \mathrm{~mL})$ and dried with anhydrous $\mathrm{MgSO}_{4}$. The solvent was evaporated and the resulting solid was washed with $n$-pentane to yield $10.7 \mathrm{~g}(92.0 \%)$ of ii as white solid.

(Chemical shifts of two isomers are reported without further assigning to syn- or anti-) ${ }^{1} \mathrm{H}$ NMR $\left(\mathrm{CDCl}_{3}, 400 \mathrm{MHz}\right): \delta$ 6.41(s, $\left.\mathrm{CHBr}_{2}\right), 6.48\left(\mathrm{~s}, \mathrm{CHBr}_{2}\right)$ 7.15, 7.19, 7.37-7.44 (m, 4H), 7.50-7.58 (m, $3 \mathrm{H}), 8.09,8.11(\mathrm{~m}, 2 \mathrm{H}) .{ }^{13} \mathrm{C}\left\{{ }^{1} \mathrm{H}\right\} \mathrm{NMR}\left(\mathrm{CDCl}_{3}, 100 \mathrm{MHz}\right): \delta 38.8,38.9\left(\mathrm{CHBr}_{2}\right.$ of syn- or anti- $)$, 124.9, 125.5 (Ar(C)-Br, syn- or anti-), 127.6, 127.7, 129.4, 129.8, 130.01, 130.05, 130.6, 130.8, 136.8, 137.0, 139.6, 139.7, 141.0. Elemental analysis calcd for $\mathrm{C}_{20} \mathrm{H}_{13} \mathrm{Br}_{5}$ (652.84): C, 36.80; H, 2.01. Found: C, 36.61; H, 1.71 .

\section{2,6-(2-CH(O) $\left.\mathrm{C}_{6} \mathrm{H}_{4}\right)_{2}-1-\mathrm{BrC}_{6} \mathrm{H}_{3}$ (iii)}

A mixture of ii $(20.00 \mathrm{~g}, 20.64 \mathrm{mmol}), \mathrm{AgNO}_{3}(21.36 \mathrm{~g}, 125.7 \mathrm{mmol})$ and $\mathrm{NaOAc}(11.33 \mathrm{~g}, 138.1$ mmol) in a solvent mixture of EtOH $(500 \mathrm{~mL})$ and THF $(100 \mathrm{~mL})$ was heated to reflux for $16 \mathrm{~h}$. After the removal of the solid by filtration the solvent was evaporated. The resulting solid was dissolved in $\mathrm{CH}_{2} \mathrm{Cl}_{2}(300 \mathrm{~mL})$ and $10 \%$ hydrochloric acid $(10 \mathrm{~mL})$ was added. This reaction mixture was stirred at room temperature for $6 \mathrm{~h}$, then washed with water $(3 \times 100 \mathrm{~mL})$ and the organic layer was dried with 
anhydrous $\mathrm{MgSO}_{4}$. The solvent was evaporated from the organic layer, and the resulting material was washed with diethyl ether and dried to yield $9.70 \mathrm{~g}$ iii as a white solid (86.7\%).

(Chemical shifts of two isomers are reported without further assigning to syn- or anti-) ${ }^{1} \mathrm{H}$ NMR $\left(\mathrm{CDCl}_{3}, 400 \mathrm{MHz}\right): \delta 9.90,9.88$ (s, CHO- of syn- or anti-), 8.02-8.05 (m, 2H), 7.66-7.72 (m, 2H), 7.55$7.59(\mathrm{~m}, 2 \mathrm{H}), 7.49-7.53(\mathrm{~m}, 1 \mathrm{H}), 7.35-7.41(\mathrm{~m}, 4 \mathrm{H}) .{ }^{13} \mathrm{C}\left\{{ }^{1} \mathrm{H}\right\} \mathrm{NMR}\left(\mathrm{CDCl}_{3}, 100 \mathrm{MHz}\right): \delta 191.3$, $191.6(\mathbf{C H O}-$ of syn- or anti-), $125.3(\mathrm{Ar}(\mathbf{C})-\mathrm{Br}), 144.6,144.4,140.4,140.3,134.1,133.89,133.92$, $133.7,131.4,131.1,131.2,130.9,128.93,128.90,128.5,127.9,127.4,127.3$. Elemental analysis calcd for $\mathrm{C}_{20} \mathrm{H}_{13} \mathrm{O}_{2} \mathrm{Br}$ (365.23): C, 65.77; H, 3.59. Found: C, 65.66; H, 3.32.

\section{2,6-(2- $\left.\mathrm{CH}_{2}(\mathrm{OH}) \mathrm{C}_{6} \mathrm{H}_{4}\right)_{2}-1-\mathrm{BrC}_{6} \mathrm{H}_{3}$ (iv)}

To a slurry of $\mathrm{NaBH}_{4}(1.20 \mathrm{~g}, 24.6 \mathrm{mmol})$ in THF $(150 \mathrm{~mL})$, a solution of iii $(9.00 \mathrm{~g}, 24.6 \mathrm{mmol})$ in MeOH (100 mL)/THF (50 mL) mixture was added slowly. After stirring overnight, 15\% hydrochloric acid $(10 \mathrm{~mL})$ was added. The mixture was stirred for another $1 \mathrm{~h}$. After removal of the solvent, diethyl ether $(200 \mathrm{~mL})$ was added, and then the solution was washed with water $(100 \mathrm{~mL} \times 3)$. The organic layer was separated and dried with anhydrous $\mathrm{MgSO}_{4}$, and then evaporated to yield $8.10 \mathrm{~g}$ iv as a white solid $(89.2 \%)$.

(Chemical shifts of two isomers are reported without further assigning to syn- or anti-) ${ }^{1} \mathrm{H}$ NMR $\left(\mathrm{CDCl}_{3}, 400 \mathrm{MHz}\right): \delta 1.59,1.72$ (broad, $\mathrm{OH}$, of $s y n$ - or anti-), 4.45, 4.48, 4.52, $4.56\left(-\mathbf{C H}_{2^{-}}\right.$, of $s y n-$ and anti-), 7.22-7.24 (m, 2H), 7.27, 7.29, 7.37-7.48 (m, 5H), 7.56-7.61 (m, 2H). ${ }^{13} \mathrm{C}\left\{{ }^{1} \mathrm{H}\right\} \mathrm{NMR}\left(\mathrm{CDCl}_{3}\right.$, $100 \mathrm{MHz}): \delta 63.3,63.4\left(-\mathrm{CH}_{2^{-}}\right.$, of $s y n-$ or $\left.a n t i-\right), 124.9,125.3(\mathrm{Ar}(\mathbf{C}) \mathrm{Br}), 127.2,127.3,127.69,127.74$, $128.0,128.1,128.6,128.7,129.6,129.9,130.2,130.4,138.6,140.5,140.7,142.4$. Elemental analysis calcd for $\mathrm{C}_{20} \mathrm{H}_{17} \mathrm{O}_{2} \mathrm{Br}$ (369.26): C, 65.05; H, 4.64. Found: C, 65.05; H, 4.52.

\section{2,6- $\left(2-\mathrm{CH}_{2} \mathrm{BrC}_{6} \mathrm{H}_{4}\right)_{2}-1-\mathrm{BrC}_{6} \mathrm{H}_{3}(\mathrm{v})$}


To a benzene $(150 \mathrm{~mL})$ solution of iv $(7.40 \mathrm{~g}, 20.0 \mathrm{mmol})$ containing $0.1 \mathrm{~mL}$ pyridine, $\operatorname{PBr}_{3}(4.95 \mathrm{~g}$, $18.3 \mathrm{mmol}$, in $10 \mathrm{~mL}$ benzene) was added slowly. The mixture was stirred for $16 \mathrm{~h}$, and then washed with water $(3 \times 100 \mathrm{~mL})$ and $5 \% \mathrm{NaHCO}_{3}(\mathrm{aq})(2 \times 100 \mathrm{~mL})$. The organic layer was collected and dried with anhydrous $\mathrm{MgSO}_{4}$. After removal of the solvent, the resulting compound was recrystallized from $n$-pentane to yield $7.83 \mathrm{~g} \mathbf{v}$ as a white solid (78.9\%).

(Chemical shifts of two isomers are reported without further assigning to syn- or anti-) ${ }^{1} \mathrm{H}$ NMR $\left(\mathrm{CDCl}_{3}, 400 \mathrm{MHz}\right): \delta$ 4.23-4.31 (m, 2H, $\left.\mathrm{CH}_{2} \mathrm{Br}\right)$, 4.44-4.47 (m, 2H, $\left.\mathrm{CH}_{2} \mathrm{Br}\right)$, 7.23-7.25 (m, 2H), 7.36$7.44(\mathrm{~m}, 6 \mathrm{H}), 7.47-7.51(\mathrm{~m}, 1 \mathrm{H}), 7.53-7.56(\mathrm{~m}, 2 \mathrm{H}) .{ }^{13} \mathrm{C}\left\{{ }^{1} \mathrm{H}\right\}$ NMR $\left(\mathrm{CDCl}_{3}, 100 \mathrm{MHz}\right): \delta 31.8,31.9$ $\left(\mathrm{CH}_{2} \mathrm{Br}\right.$, of syn- or anti-), 127.08, $127.11(\mathrm{Ar}(\mathbf{C})-\mathrm{Br}$, of syn- or anti-), 128.5, 128.6, 128.7, 128.87, $128.89,130.4,130.58,130.62,130.73,130.82,135.6,135.7,141.58,141.66,141.68$. Elemental analysis calcd for $\mathrm{C}_{20} \mathrm{H}_{15} \mathrm{Br}_{3}$ (495.05): C, 48.52; H, 3.05. Found: C, 48.67; H, 2.83.

\section{2,6- $\left(2-\mathrm{CH}_{2} \mathrm{PPh}_{2} \mathrm{C}_{6} \mathrm{H}_{4}\right)_{2}-1-\mathrm{BrC}_{6} \mathrm{H}_{3}(1 \mathrm{a})$}

Freshly cut $\mathrm{Li}(0.5 \mathrm{~g}, 71 \mathrm{mmol})$ was added to a solution of diphenylchlorophosphine $(2.00 \mathrm{~g}, 9.06$ mmol) in anhydrous THF (100 mL). The mixture was stirred for $4 \mathrm{~h}$ at room temperature. The excess Li metal was removed, and the resulting dark red solution was cooled to $-78^{\circ} \mathrm{C}$. To the chilled solution was added a solution of $\mathbf{v}(2.00 \mathrm{~g}, 4.04 \mathrm{mmol})$ in anhydrous THF (40 mL). The reaction mixture was warmed to room temperature and stirred for an additional $16 \mathrm{~h}$. Solvent was removed in vacuo and the remaining solid was dissolved in $50 \mathrm{~mL}$ diethyl ether. The undissolved solid was removed by filtration. Removal of the solvent in the filtrate yielded $2.62 \mathrm{~g} \mathbf{1 a}$ as white solid (91.9\%).

(Chemical shifts of two isomers are reported without further assigning to syn- or anti-) ${ }^{1} \mathrm{H}$ NMR $\left(\mathrm{CDCl}_{3}, 400 \mathrm{MHz}\right): \delta 3.14-3.20\left(\mathrm{~m}, 2 \mathrm{H},-\mathrm{CH}_{2^{-}}\right), 3.23-3.38\left(\mathrm{~m}, 2 \mathrm{H},-\mathrm{CH}_{2^{-}}\right), 6.79\left(\mathrm{~d}, J_{\mathrm{HH}}=8 \mathrm{~Hz}, 1 \mathrm{H}\right)$, 6.94-7.35 (m, 30H). ${ }^{31} \mathrm{P}$ NMR $\left(\mathrm{CDCl}_{3}, 162 \mathrm{MHz}\right): \delta-10.3,-10.2$ (syn- or anti-). An air-stable derivative $\left[2,6-\left(2-\mathrm{CH}_{2} \mathrm{P}(=\mathrm{O}) \mathrm{Ph}_{2} \mathrm{C}_{6} \mathrm{H}_{4}\right)_{2}-1-\mathrm{BrC}_{6} \mathrm{H}_{3}\right]$ for analysis was produced by dissolving a sample of 
1a in THF and bubbling dioxygen through the solution, followed by evaporation. Elemental analysis calcd for $\left[(\mathbf{1 a})(\mathrm{O})_{2}\right], \mathrm{C}_{44} \mathrm{H}_{35} \mathrm{BrP}_{2} \mathrm{O}_{2}$ (737.61): C, 71.65; H, 4.78. Found: C, 70.72; H, 5.16.

\section{2,6- $\left(2-\mathrm{CH}_{2} \mathrm{PCy}_{2} \mathrm{C}_{6} \mathrm{H}_{4}\right)_{2}-1-\mathrm{BrC}_{6} \mathrm{H}_{3}(1 \mathrm{~b})$}

To a mixture of $\mathbf{v}(1.06 \mathrm{~g}, 2.14 \mathrm{mmol})$ and dicyclohexylphosphine $(0.95 \mathrm{~g}, 4.8 \mathrm{mmol}), 20 \mathrm{~mL}$ anhydrous acetone was added, and the resultant mixture was heated to reflux overnight under nitrogen. After cooling to room temperature, the solvent was removed in vacuo. The remaining material was dissolved in $50 \mathrm{~mL}$ of anhydrous THF, and sodium acetate $(0.70 \mathrm{~g}, 8.5 \mathrm{mmol})$ was added. The mixture was stirred overnight. Excess sodium acetate was then removed by filtration and then the solvent was removed in vacuo. The remaining waxy compound was washed with small amount of hexanes, and dried in vacuo to yield $\mathbf{1 b}$ as a white fine powder $(0.97 \mathrm{~g}, 62 \%)$.

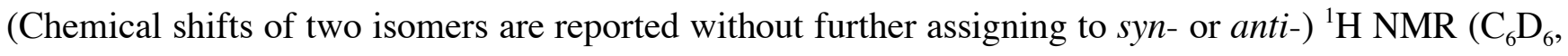
400 MHz): $\delta$ 0.90-1.90 (m, 42H, Cyclohexyl), 2.87 (m, 2H, P-Cy(H)), $2.83\left(\mathrm{~m}, 2 \mathrm{H},-\mathbf{C H}_{2^{-}}\right), 2.98(\mathrm{~d}$, $\left.J_{\mathrm{HH}}=15 \mathrm{~Hz}, 2 \mathrm{H},-\mathrm{CH}_{2^{-}}\right), 7.07-7.25(\mathrm{~m}, 8 \mathrm{H}), 7.36(\mathrm{~m}, 1 \mathrm{H}), 7.87\left(\mathrm{~d}, J_{\mathrm{HH}}=8 \mathrm{~Hz}, 2 \mathrm{H}\right) .{ }^{31} \mathrm{P} \mathrm{NMR}\left(\mathrm{CDCl}_{3}\right.$, $162 \mathrm{MHz}): \delta 1.3$.

\section{$\left[2,6-\left(2-\mathrm{CH}_{2} \mathbf{P}^{\mathrm{t}} \mathrm{Bu}_{2} \mathrm{C}_{6} \mathrm{H}_{4}\right)_{2}-1-\mathrm{BrC}_{6} \mathrm{H}_{3}\right](\mathrm{HBr})_{2}\left(1 c^{\prime}\right)$}

A solution of $\mathbf{v}(1.50 \mathrm{~g}, 3.03 \mathrm{mmol})$ in anhydrous acetone $(20 \mathrm{~mL})$ was added to $1.00 \mathrm{~g}(6.84 \mathrm{mmol})$ of di-t-butylphosphine, and the resultant mixture was heated to reflux overnight under nitrogen. After cooling to room temperature, $2.35 \mathrm{~g}$ 1c' as white solid compound (98.5\%) was obtained upon removal of the solvent.

${ }^{1} \mathrm{H}$ NMR $\left(\mathrm{CD}_{2} \mathrm{Cl}_{2}, 400 \mathrm{MHz}\right): \delta 1.33\left(\mathrm{~d}, J_{\mathrm{HH}}=16 \mathrm{~Hz}, 18 \mathrm{H},-\mathrm{C}\left(\mathrm{CH}_{3}\right)_{3}\right), \delta 1.48\left(\mathrm{~d}, J_{\mathrm{HH}}=16 \mathrm{~Hz}, 18 \mathrm{H},-\right.$ $\left.\mathrm{C}\left(\mathrm{CH}_{3}\right)_{3}\right), 3.55\left(\mathrm{~m}, 2 \mathrm{H},-\mathrm{CH}_{2^{-}}\right), 3.82\left(\mathrm{~m}, 2 \mathrm{H},-\mathrm{CH}_{2^{-}}\right), 7.18\left(\mathrm{~d}, J_{\mathrm{HH}}=7 \mathrm{~Hz}, 2 \mathrm{H}\right), 7.47\left(\mathrm{t}, J_{\mathrm{HH}}=7 \mathrm{~Hz}, 2 \mathrm{H}\right)$, $7.53(\mathrm{~m}, 2 \mathrm{H}), 7.73\left(\mathrm{~d}, J_{\mathrm{HH}}=8 \mathrm{~Hz}, 2 \mathrm{H}\right), 7.89(\mathrm{~m}, 1 \mathrm{H}), 8.01\left(\mathrm{~d}, J_{\mathrm{HH}}=8 \mathrm{~Hz}, 2 \mathrm{H}\right), 8.54$ (doublet of virtual 
triplets, $\left.J_{\mathrm{PH}}=486 \mathrm{~Hz}, 2 \mathrm{H}\right) .{ }^{13} \mathrm{C}\left\{{ }^{1} \mathrm{H}\right\} \mathrm{NMR}\left(\mathrm{CD}_{2} \mathrm{Cl}_{2}, 100 \mathrm{MHz}\right): \delta 20.1\left(\mathrm{~d}, J_{\mathrm{PC}}=37 \mathrm{~Hz},-\mathrm{CH}_{2}-\right), 27.7(\mathrm{~s}$, $\left.-\mathrm{C}\left(\mathrm{CH}_{3}\right)_{3}\right), 27.9\left(\mathrm{~s},-\mathrm{C}\left(\mathrm{CH}_{3}\right)_{3}\right), 33.6\left(\mathrm{~d}, J_{\mathrm{PC}}=32 \mathrm{~Hz},-\mathbf{C}\left(\mathrm{CH}_{3}\right)_{3}\right), 33.9\left(\mathrm{~d}, J_{\mathrm{PC}}=33 \mathrm{~Hz},-\mathbf{C}\left(\mathrm{CH}_{3}\right)_{3}\right), 124.8$, $128.7\left(\mathrm{~d}, J_{\mathrm{PC}}=8 \mathrm{~Hz}\right), 128.9\left(\mathrm{~d}, J_{\mathrm{PC}}=2 \mathrm{~Hz}\right), 129.7,130.5,131.0,131.9\left(\mathrm{~d}, J_{\mathrm{PC}}=5 \mathrm{~Hz}\right), 133.0,141.2(\mathrm{~d}$, $\left.J_{\mathrm{PC}}=5 \mathrm{~Hz}\right), 141.7 .{ }^{31} \mathrm{P} \mathrm{NMR}\left(\mathrm{CD}_{2} \mathrm{Cl}_{2}, 162 \mathrm{MHz}\right): \delta 32.3\left(\mathrm{~d}, J_{\mathrm{PH}}=480 \mathrm{~Hz}\right)$. Elemental analysis calcd for $\mathrm{C}_{36} \mathrm{H}_{53} \mathrm{Br}_{3} \mathrm{P}_{2}$ (787.48): C, 54.91; H, 6.78. Found: C, 54.64; H, 6.81.

\section{2,6-(2- $\left.\mathrm{CH}_{2} \mathrm{P}^{t} \mathrm{Bu}_{2} \mathrm{C}_{6} \mathrm{H}_{4}\right)_{2}-1-\mathrm{BrC}_{6} \mathrm{H}_{3}(1 \mathrm{c})$}

To a solution of 1' $(1.00 \mathrm{~g}, 1.27 \mathrm{mmol})$ in THF $(50 \mathrm{~mL})$, sodium acetate $(0.40 \mathrm{~g}, 4.9 \mathrm{mmol})$ was added and the mixture was stirred for overnight at room temperature. The excess sodium acetate was removed by filtration. After removal of the solvent, the remaining waxy material was washed by small amount of hexanes, and $0.58 \mathrm{~g} \mathbf{1 c}$ as white fine powder compound was obtained after drying (73\%).

${ }^{1} \mathrm{H} \mathrm{NMR}\left(\mathrm{CDCl}_{3}, 400 \mathrm{MHz}\right): \delta 0.94\left(\mathrm{~d}, J_{\mathrm{HH}}=11 \mathrm{~Hz}, 18 \mathrm{H},-\mathrm{C}\left(\mathrm{CH}_{3}\right)_{3}\right.$, anti- $), \delta 1.07\left(\mathrm{~d}, J_{\mathrm{HH}}=11 \mathrm{~Hz}\right.$, $18 \mathrm{H},-\mathrm{C}\left(\mathrm{CH}_{3}\right)_{3}$, anti-), [Note: due to some overlapping signals for anti- and syn- isomersm anti- : syn$=10: 1$; the following spectroscopy data of only the major (the presumed anti- form) are reported. Signals at $\delta 1.00\left(\mathrm{~d}, J_{\mathrm{HH}}=11 \mathrm{~Hz},-\mathrm{C}\left(\mathrm{CH}_{3}\right)_{3}\right.$, syn- $), 1.12\left(\mathrm{~d}, J_{\mathrm{HH}}=11 \mathrm{~Hz},-\mathrm{C}\left(\mathrm{CH}_{3}\right)_{3}\right.$, syn-) can be clearly seen, however]. $2.67\left(\mathrm{~m}, 2 \mathrm{H},-\mathrm{CH}_{2^{-}}\right), 2.80\left(\mathrm{~m}, 2 \mathrm{H},-\mathrm{CH}_{2^{-}}\right), 7.09\left(\mathrm{~d}, J_{\mathrm{HH}}=7 \mathrm{~Hz}, 2 \mathrm{H}\right), 7.22\left(\mathrm{t}, J_{\mathrm{HH}}=7\right.$ $\mathrm{Hz}, 2 \mathrm{H}), 7.26-7.34(\mathrm{~m}, 4 \mathrm{H}), 7.41(\mathrm{~m}, 1 \mathrm{H}), 7.73\left(\mathrm{~d}, J_{\mathrm{HH}}=8 \mathrm{~Hz}, 2 \mathrm{H}\right) .{ }^{13} \mathrm{C}\left\{{ }^{1} \mathrm{H}\right\} \mathrm{NMR}\left(\mathrm{CDCl}_{3}, 100\right.$ MHz): $\delta 25.9\left(\mathrm{~d}, J_{\mathrm{PC}}=24 \mathrm{~Hz},-\mathbf{C H}_{2}-\right), 29.8\left(\mathrm{~d}, J_{\mathrm{PC}}=13 \mathrm{~Hz},-\mathrm{C}\left(\mathbf{C H}_{3}\right)_{3}\right), 30.0\left(\mathrm{~d} J_{\mathrm{PC}}=13 \mathrm{~Hz},-\mathrm{C}\left(\mathbf{C H}_{3}\right)_{3}\right)$, $31.8\left(\mathrm{~d}, J_{\mathrm{PC}}=16 \mathrm{~Hz},-\mathbf{C}\left(\mathrm{CH}_{3}\right)_{3}\right), 32.0\left(\mathrm{~d}, J_{\mathrm{PC}}=17 \mathrm{~Hz},-\mathbf{C}\left(\mathrm{CH}_{3}\right)_{3}\right), 125.4\left(\mathrm{~d}, J_{\mathrm{PC}}=2 \mathrm{~Hz}\right), 126.6(\mathrm{~s}), 127.8$ $(\mathrm{s}), 129.8(\mathrm{~s}), 130.6(\mathrm{~s}), 130.7(\mathrm{~s}), 131.1(\mathrm{~s}), 139.5\left(\mathrm{~d}, J_{\mathrm{PC}}=12 \mathrm{~Hz}\right), 141.8\left(\mathrm{~d}, J_{\mathrm{PC}}=4 \mathrm{~Hz}\right), 143.2(\mathrm{~s}) .{ }^{31} \mathrm{P}$ NMR (CDCl, $162 \mathrm{MHz}): \delta 30.4$ (s), 32.4 (s), (anti- : syn- 10:1).

\section{$\left[2,6-\left(2-\mathrm{CH}_{2} \mathrm{PPh}_{2} \mathrm{C}_{6} \mathrm{H}_{4}\right)_{2} \mathrm{C}_{6} \mathrm{H}_{3} \mathrm{PdBr}\right](3 \mathrm{a})$}

A mixture of $1 \mathrm{a}(0.80 \mathrm{~g}, 1.1 \mathrm{mmol})$ and $\mathrm{Pd}_{2}(\mathrm{dba})_{3}(0.50 \mathrm{~g}, 0.55 \mathrm{mmol})$ were dissolved in anhydrous benzene $(20 \mathrm{~mL})$, and stirred at room temperature for overnight. The resultant solution was filtered and 
the solvent was removed in vacuo. The crude mixture was chromatographed over silica gel using ethyl acetate/hexanes (1:4 v/v) as eluent to afford $0.40 \mathrm{~g} \mathbf{3 a}$ as pale yellow crystalline solid (45\%).

${ }^{1} \mathrm{H}$ NMR $\left(\mathrm{CDCl}_{3}, 600 \mathrm{MHz}\right.$ ): $\delta 2.91$ (doublet of virtual triplets, $J_{\mathrm{HH}}=13 \mathrm{~Hz}, 2 \mathrm{H},-\mathrm{CH}_{2}-$ ), 3.21 (doublet of virtual triplets, $\left.J_{\mathrm{HH}}=13 \mathrm{~Hz}, 2 \mathrm{H},-\mathrm{CH}_{2^{-}}\right), 6.38(\mathrm{~m}, 2 \mathrm{H}), 6.65\left(\mathrm{~d}, J_{\mathrm{HH}}=7 \mathrm{~Hz}, 2 \mathrm{H}\right), 6.73\left(\mathrm{~d}, J_{\mathrm{HH}}=7 \mathrm{~Hz}\right.$, $2 \mathrm{H}), 7.02\left(\mathrm{t}, J_{\mathrm{HH}}=7 \mathrm{~Hz}, 1 \mathrm{H}\right), 7.10-7.15(\mathrm{~m}, 8 \mathrm{H}), 7.20\left(\mathrm{t}, J_{\mathrm{HH}}=7 \mathrm{~Hz}, 4 \mathrm{H}\right), 7.28\left(\mathrm{t}, J_{\mathrm{HH}}=7 \mathrm{~Hz}, 2 \mathrm{H}\right), 7.45$ $\left(\mathrm{t}, J_{\mathrm{HH}}=7 \mathrm{~Hz}, 4 \mathrm{H}\right), 7.57\left(\mathrm{t}, J_{\mathrm{HH}}=7 \mathrm{~Hz}, 2 \mathrm{H}\right), 7.58-7.61(\mathrm{~m}, 4 \mathrm{H}) .{ }^{13} \mathrm{C}\left\{{ }^{1} \mathrm{H}\right\} \mathrm{NMR}\left(\mathrm{CDCl}_{3}, 150 \mathrm{MHz}\right): \delta$ 32.1 (virtual triplet, $\left.J_{\mathrm{PC}}=13 \mathrm{~Hz},-\mathrm{CH}_{2}-\right), 124.9(\mathrm{~s}), 126.4(\mathrm{~s}), 127.3(\mathrm{~m}), 129.0$ (virtual triplet, $J_{\mathrm{PC}}=5$ Hz), $129.5(\mathrm{~s}), 129.6(\mathrm{~m}), 130.0(\mathrm{~s}), 130.6(\mathrm{~s}), 131.0$ (virtual triplet, $\left.J_{\mathrm{PC}}=20 \mathrm{~Hz}\right), 132.0(\mathrm{~s}), 133.6$ (virtual triplet, $J_{\mathrm{PC}}=22 \mathrm{~Hz}$ ), 133.70 (virtual triplet, $J_{\mathrm{PC}}=7 \mathrm{~Hz}$ ), 133.84 (virtual triplet, $J_{\mathrm{PC}}=5 \mathrm{~Hz}$ ), 145.0 (virtual triplet, $J_{\mathrm{PC}}=3 \mathrm{~Hz}$ ), $146.7\left(\mathrm{~s}, \operatorname{Ar}(\mathbf{C})-\mathrm{Pd}\right.$-), 147.3 (virtual triplet, $J_{\mathrm{PC}}=4 \mathrm{~Hz}$ ). ${ }^{31} \mathrm{P}$ NMR $\left(\mathrm{CDCl}_{3}, 162 \mathrm{MHz}\right): \delta 26.3$ (s). Elemental analysis calcd for $\mathrm{C}_{44} \mathrm{H}_{35} \mathrm{BrP}_{2} \mathrm{Pd}$ (812.03): C, 65.08; H, 4.34. Found: C, 64.90; H, 4.13.

\section{$\left[2,6-\left(2-\mathrm{CH}_{2} \mathrm{PCy}_{2} \mathrm{C}_{6} \mathrm{H}_{4}\right)_{2} \mathrm{C}_{6} \mathrm{H}_{3} \mathrm{PdBr}\right](3 \mathrm{~b})$}

To a mixture of $\mathbf{1 b}(0.30 \mathrm{~g}, 0.42 \mathrm{mmol})$ and $\mathrm{Pd}_{2}(\mathrm{dba})_{3}(0.23 \mathrm{~g}, 0.26 \mathrm{mmol}), 15 \mathrm{~mL}$ benzene was added, and mixture was stirred overnight at room temperature. The resultant solution was filtered and solvent was removed in vacuo. The crude product was chromatographed over silica gel using ethyl acetate/hexanes $(1: 10 \mathrm{v} / \mathrm{v})$ as eluent to afford $0.16 \mathrm{~g} \mathbf{3 b}$ as pale yellow crystalline solid (47\%). Analytical pure product was obtained via recrystallization from $\mathrm{CHCl}_{3} /$ hexanes.

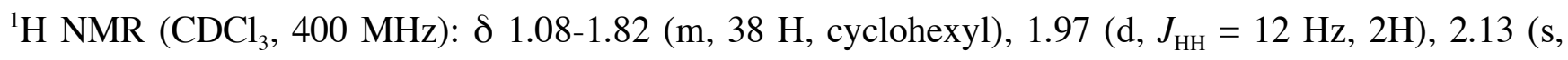
$2 \mathrm{H}$ ), $2.79(\mathrm{~m}, 2 \mathrm{H}), 2.53$ (doublet of virtual triplets, $J_{\mathrm{HH}}=13 \mathrm{~Hz}, 2 \mathrm{H},-\mathrm{CH}_{2^{-}}$), 2.84 (doublet of virtual triplets, $\left.J_{\mathrm{HH}}=13 \mathrm{~Hz}, 2 \mathrm{H},-\mathrm{CH}_{2^{-}}\right), 6.75\left(\mathrm{~d}, J_{\mathrm{HH}}=8 \mathrm{~Hz}, 2 \mathrm{H}\right), 7.04\left(\mathrm{t}, J_{\mathrm{HH}}=8 \mathrm{~Hz}, 1 \mathrm{H}\right), 7.22-7.25(\mathrm{~m}, 4 \mathrm{H})$, 7.27-7.31 (m, 2H), $7.34\left(\mathrm{~d}, J_{\mathrm{HH}}=7 \mathrm{~Hz}, 2 \mathrm{H}\right) .{ }^{13} \mathrm{C}\left\{{ }^{1} \mathrm{H}\right\} \mathrm{NMR}\left(\mathrm{CDCl}_{3}, 100 \mathrm{MHz}\right): \delta 23.7$ (virtual triplet, $\left.J_{\mathrm{PC}}=10 \mathrm{~Hz},-\mathrm{CH}_{2}-\right), 26.0(\mathrm{~s}), 26.9(\mathrm{~s}), 27.1\left(\right.$ virtual triplet, $\left.J_{\mathrm{PC}}=6 \mathrm{~Hz}\right), 27.3-27.7(\mathrm{~m}), 28.5(\mathrm{~s}), 28.8(\mathrm{~s})$, 
$28.9(\mathrm{~s}), 30.4(\mathrm{~s}), 35.2$ (virtual triplet, $J_{\mathrm{PC}}=10 \mathrm{~Hz}$ ), 36.3 (virtual triplet, $\left.J_{\mathrm{PC}}=10 \mathrm{~Hz}\right), 124.1(\mathrm{~s}), 126.4$ $(\mathrm{s}), 126.6(\mathrm{~s}) 128.6(\mathrm{~s}), 129.9(\mathrm{~s}), 130.2(\mathrm{~s}), 134.3(\mathrm{~s}), 146.0$ (virtual triplet, $\left.J_{\mathrm{PC}}=3 \mathrm{~Hz}\right), 147.4$ (m, upon analysis of 2-D NMR (H-COSY, HMQC and HMBC), the carbon resonance of $\operatorname{Ar}(\mathbf{C})-\mathrm{Pd}$ is overlapping with the virtual triplet at $147.4 \mathrm{ppm}) .{ }^{31} \mathrm{P} \mathrm{NMR}\left(\mathrm{CDCl}_{3}, 162 \mathrm{MHz}\right): \delta 33.7$ (s). Elemental analysis calcd for $\mathrm{C}_{44} \mathrm{H}_{59} \mathrm{BrP}_{2} \mathrm{Pd} \cdot \mathrm{CHCl}_{3}$ : C, 56.56; H, 6.33. Found: C, 56.68; H, 6.22.

\section{$\left[2,6-\left(2-\mathrm{CH}_{2} \mathrm{P}^{t} \mathrm{Bu}_{2} \mathrm{C}_{6} \mathrm{H}_{4}\right)_{2} \mathrm{C}_{6} \mathrm{H}_{3} \mathrm{PdBr}\right](3 \mathrm{c})$}

To a mixture of $1 \mathrm{c}(0.30 \mathrm{~g}, 0.48 \mathrm{mmol})$ and $\mathrm{Pd}_{2}(\mathrm{dba})_{3}(0.27 \mathrm{~g}, 0.29 \mathrm{mmol}), 25 \mathrm{~mL}$ benzene was added, and the mixture was stirred overnight at room temperature. The resultant solution was filtered and the solvent was removed in vacuo. The crude product was chromatographed over silica gel using ethyl acetate/hexanes $(1: 4 \mathrm{v} / \mathrm{v})$ as eluent to afford $0.23 \mathrm{~g} \mathrm{3c}$ as pale yellow crystalline solid (66\%). Analytical pure product was obtained via recrystallization from $\mathrm{CHCl}_{3} /$ hexanes.

${ }^{1} \mathrm{H} \mathrm{NMR}\left(\mathrm{CDCl}_{3}, 400 \mathrm{MHz}\right): \delta 1.29$ (virtual triplet, $\left.J_{\mathrm{PH}}=7 \mathrm{~Hz} 18 \mathrm{H},-\mathrm{C}\left(\mathrm{CH}_{3}\right)_{3}\right), 1.32($ broad, 6H, $\left.\mathrm{C}\left(\mathrm{CH}_{3}\right)_{3}\right), 1.59$ (broad, 6H, $\left.-\mathrm{C}\left(\mathrm{CH}_{3}\right)_{3}\right), 1.84$ (broad, 6H, $\left.-\mathrm{C}\left(\mathrm{CH}_{3}\right)_{3}\right), 2.69$ (doublet of virtual triplets, $J_{\mathrm{HH}}$ $\left.=13 \mathrm{~Hz}, 2 \mathrm{H},-\mathrm{CH}_{2^{-}}\right), 2.93$ (doublet of virtual triplets, $\left.J_{\mathrm{HH}}=13 \mathrm{~Hz}, 2 \mathrm{H},-\mathrm{CH}_{2^{-}}\right), 6.58\left(\mathrm{~d}, J_{\mathrm{HH}}=8 \mathrm{~Hz}, 2 \mathrm{H}\right)$, $6.94\left(\mathrm{t}, J_{\mathrm{HH}}=8 \mathrm{~Hz}, 1 \mathrm{H}\right), 7.22(\mathrm{~m}, 2 \mathrm{H}), 7.32(\mathrm{~m}, 4 \mathrm{H}), 7.37(\mathrm{~m}, 2 \mathrm{H}) . \quad{ }^{13} \mathrm{C}\left\{{ }^{1} \mathrm{H}\right\} \mathrm{NMR}\left(\mathrm{CDCl}_{3}, 100\right.$ $\mathrm{MHz}$ ): $\delta 23.7$ (virtual triplet, $J_{\mathrm{PC}}=10 \mathrm{~Hz},-\mathbf{C H}_{2}-$ ), 31.3 (virtual triplet, $\left.J_{\mathrm{PC}}=2 \mathrm{~Hz},-\mathrm{C}\left(\mathbf{C H}_{3}\right)_{3}\right), 32.2$ (broad, $\left.-\mathrm{C}\left(\mathrm{CH}_{3}\right)_{3}\right), 36.4$ (virtual triplet, $\left.J_{\mathrm{PC}}=5 \mathrm{~Hz},-\mathbf{C}\left(\mathrm{CH}_{3}\right)_{3}\right), 38.8$ (virtual triplet, $J_{\mathrm{PC}}=7 \mathrm{~Hz},-$ $\left.\mathbf{C}\left(\mathrm{CH}_{3}\right)_{3}\right), 124.1$ (s), 126.1 (s), 126.9 (s), 129.3 (s), 129.5 (s), 130.2 (s), 134.5 (s), 146.3 (virtual triplet, $\left.J_{\mathrm{PC}}=3 \mathrm{~Hz}\right), 147.0(\mathrm{~s}, \operatorname{Ar}(\mathbf{C})-\mathrm{Pd}), 148.6$ (virtual triplet, $\left.J_{\mathrm{PC}}=3 \mathrm{~Hz}\right) .{ }^{31} \mathrm{P} \mathrm{NMR}\left(\mathrm{CDCl}_{3}, 162 \mathrm{MHz}\right): \delta$ 55.1 (s). Elemental analysis calcd for $\mathrm{C}_{36} \mathrm{H}_{52} \mathrm{BrP}_{2} \mathrm{Pd} \cdot 1 / 3 \mathrm{CHCl}_{3}: \mathrm{C}, 56.54 ; \mathrm{H}, 6.70$. Found: C, 56.41; H, 6.67.

\section{[2,6- $\left.\left(2-\mathrm{CH}_{2} \mathrm{PPh}_{2} \mathrm{C}_{6} \mathrm{H}_{4}\right)_{2} \mathrm{C}_{6} \mathrm{H}_{3} \mathrm{NiBr}\right](4 \mathrm{a})$}


A mixture of $1 \mathrm{a}(0.070 \mathrm{~g}, 0.099 \mathrm{mmol})$ and $\mathrm{Ni}(\mathrm{COD})_{2}(0.026 \mathrm{~g}, 0.095 \mathrm{mmol})$ were dissolved in anhydrous benzene $(10 \mathrm{~mL})$, and stirred at room temperature for overnight. The resultant solution was filtered and solvent was removed in vacuo. The crude product was chromatographed over silica gel using ethyl acetate/hexanes (1:4 v/v) as eluent to afford $0.040 \mathrm{~g} \mathbf{4 a}$ as pale yellow crystalline solid $(55 \%)$.

${ }^{1} \mathrm{H} \mathrm{NMR}\left(\mathrm{CDCl}_{3}, 400 \mathrm{MHz}\right): \delta 2.71$ (doublet of virtual triplets, $J_{\mathrm{HH}}=12 \mathrm{~Hz}, 2 \mathrm{H},-\mathrm{CH}_{2^{-}}$), 3.04 (doublet of virtual triplets, $\left.J_{\mathrm{HH}}=12 \mathrm{~Hz}, 2 \mathrm{H},-\mathrm{CH}_{2}-\right), 6.26(\mathrm{~m}, 2 \mathrm{H}), 6.58\left(\mathrm{~d}, J_{\mathrm{HH}}=7 \mathrm{~Hz}, 2 \mathrm{H}\right), 6.68\left(\mathrm{~d}, J_{\mathrm{HH}}=7 \mathrm{~Hz}\right.$, $2 \mathrm{H}), 6.94\left(\mathrm{t}, J_{\mathrm{HH}}=7 \mathrm{~Hz}, 1 \mathrm{H}\right), 7.07-7.18(\mathrm{~m}, 12 \mathrm{H}), 7.25\left(\mathrm{t}, J_{\mathrm{HH}}=7 \mathrm{~Hz}, 2 \mathrm{H}\right), 7.46\left(\mathrm{t}, J_{\mathrm{HH}}=7 \mathrm{~Hz}, 4 \mathrm{H}\right)$,

7.57-7.65 (m, 6H). ${ }^{13} \mathrm{C}\left\{{ }^{1} \mathrm{H}\right\} \mathrm{NMR}\left(\mathrm{CDCl}_{3}, 150 \mathrm{MHz}\right): \delta 31.6$ (virtual triplet, $J_{\mathrm{PC}}=13 \mathrm{~Hz},-\mathrm{CH}_{2^{-}}$), $124.5(\mathrm{~s}), 126.3(\mathrm{~s}), 126.6(\mathrm{~m}), 127.2$ (virtual triplet, $\left.J_{\mathrm{PC}}=5 \mathrm{~Hz}\right), 127.5(\mathrm{~s}), 129.0$ (virtual triplet, $J_{\mathrm{PC}}=5$ $\mathrm{Hz}$ ), $129.3(\mathrm{~s}), 129.4(\mathrm{~m}), 129.5(\mathrm{~s}), 130.3(\mathrm{~s}), 131.0$ (virtual triplet, $J_{\mathrm{PC}}=18 \mathrm{~Hz}$ ), 132.1 (s), 133.2 (virtual triplet, $J_{\mathrm{PC}}=6 \mathrm{~Hz}$ ), 133.7 (virtual triplet, $J_{\mathrm{PC}}=5 \mathrm{~Hz}$ ), 134.8 (virtual triplet, $J_{\mathrm{PC}}=20 \mathrm{~Hz}$ ), 144.7 (s), 145.6 (virtual triplet, $J_{\mathrm{PC}}=33 \mathrm{~Hz}, \operatorname{Ar}(\mathbf{C})-\mathrm{Ni}$ ), 149.1 (virtual triplet, $J_{\mathrm{PC}}=3 \mathrm{~Hz}$ ). ${ }^{31} \mathrm{P} \mathrm{NMR}\left(\mathrm{CDCl}_{3}\right.$, $162 \mathrm{MHz}): \delta 27.0(\mathrm{~s})$.

\section{X-ray Crystallographic Study:}

X-ray quality crystal (a colorless block of approximate dimensions $0.45 \times 0.30 \times 0.29 \mathrm{~mm}^{3}$ ) was grown by slow diffusion of hexanes into a chloroform solution of 3a. The X-ray intensity data were measured at $300 \mathrm{~K}$ on a Bruker SMART $1000 \mathrm{CCD}$-based X-ray diffractometer system equipped with a Motarget X-ray tube $(\lambda=0.71073 \AA)$ operated at 2000 watts power. The detector was placed at a distance of $5.020 \mathrm{~cm}$ from the crystal. Data were measured using omega scans of $0.3^{\circ}$ per frame for 5 seconds such that a hemisphere was collected. A total of 1271 frames were collected with a final resolution of $0.75 \AA$. No decay was indicated by the recollection of the first 50 frames at the end of data collection. 
The frames were integrated with the Bruker SAINT ${ }^{\odot}$ software package using a narrow-frame integration algorithm, which also corrects for the Lorentz and polarization effects. Absorption corrections were applied using SADABS supplied by George Sheldrick.

The structure was solved and refined in the space group $C 2 / \mathrm{c}$ (No. 15) using the Bruker $\mathrm{SHELXTL}^{\odot}$ (Version 5.1) Software. The positions of all non-hydrogen atoms were derived from the Patterson solution. The asymmetric unit consists of a half of the molecule and is related to the other half via a crystallographic 2-fold axis that is coincidental with the $\mathrm{C} 1-\mathrm{Pd}-\mathrm{Br}$ vector. With all non-hydrogen atoms being anisotropic and all hydrogen atoms being isotropic the structure was refined to convergence by least squares method on $\mathrm{F}^{2}$, SHELXL-93, incorporated in SHELXTL.PC V 5.03. 


\section{Variable Temperature NMR spectroscopy}

In order to assess the structural rigidity of the pincer platform, the ${ }^{1} \mathrm{H}$ NMR of $\mathbf{3 a}$ was investigated in detail. For a static or slowly exchanging (between atropisomers a and $\mathbf{a}^{\prime}$ ) structure, the two diastereotopic benzyl protons of some previously reported pincer complexes can be resolved, and at increased temperatures show coalescence phenomena indicative of fast exchange between atropisomers. Our studies of 3a have determined that the ${ }^{1} \mathrm{H}$ NMR signals for the benzyl protons are both highly temperature and solvent dependent in a complicated manner. In $\mathrm{CD}_{2} \mathrm{Cl}_{2}$ or $\mathrm{CDCl}_{2} \mathrm{CDCl}_{2}$, the two sets of benzyl protons are well separated (a pair of virtual triplets for each signal, Figure S9) at room temperature. At higher temperatures up to $130^{\circ} \mathrm{C}$ in $\mathrm{CDCl}_{2} \mathrm{CDCl}_{2}$, the signals show no evidence for exchange, and, in fact, seem to move further apart. Cooling a solution of $\mathbf{3 a}$ in $\mathrm{CD}_{2} \mathrm{Cl}_{2}$ can bring about a "phantom" coalescence temperature of about $-75^{\circ} \mathrm{C}$. Similar experiments on compound $3 \mathbf{c}$ in dmso- $\mathrm{d}_{6}$ also reveals a "phantom" coalescence temperature of about $100^{\circ} \mathrm{C}$, above which these signals remain as an unresolved broad signal. Since the analysis of the benzyl protons can be unreliable and unpredictable, analysis of the ${ }^{1} \mathrm{H}$ NMR data for the two $\mathrm{Ph}$ groups on the phosphorus atoms was carried out. At all temperature the sets of protons corresponding to the $\mathrm{Ph}$ groups proximal and distal to the $\mathrm{Br}$ atom showed no evidence for exchange.

For the potential risks associated with assessing the degree of flexibility of ring systems where there may also be a inherent temperature dependence, as well a solvent dependence, of key NMR resonances

see: (a) Mislow, K.; Glass, M. A. W.; Hopps, H. B.; Simon, E.; Jr Wahl, G. H. J. Am. Chem. Soc. 1962, 86, 1710. (b) Sutherland, I. O.; Ramsay, M. V. Tetrahedron 1965, 21, 3401. 


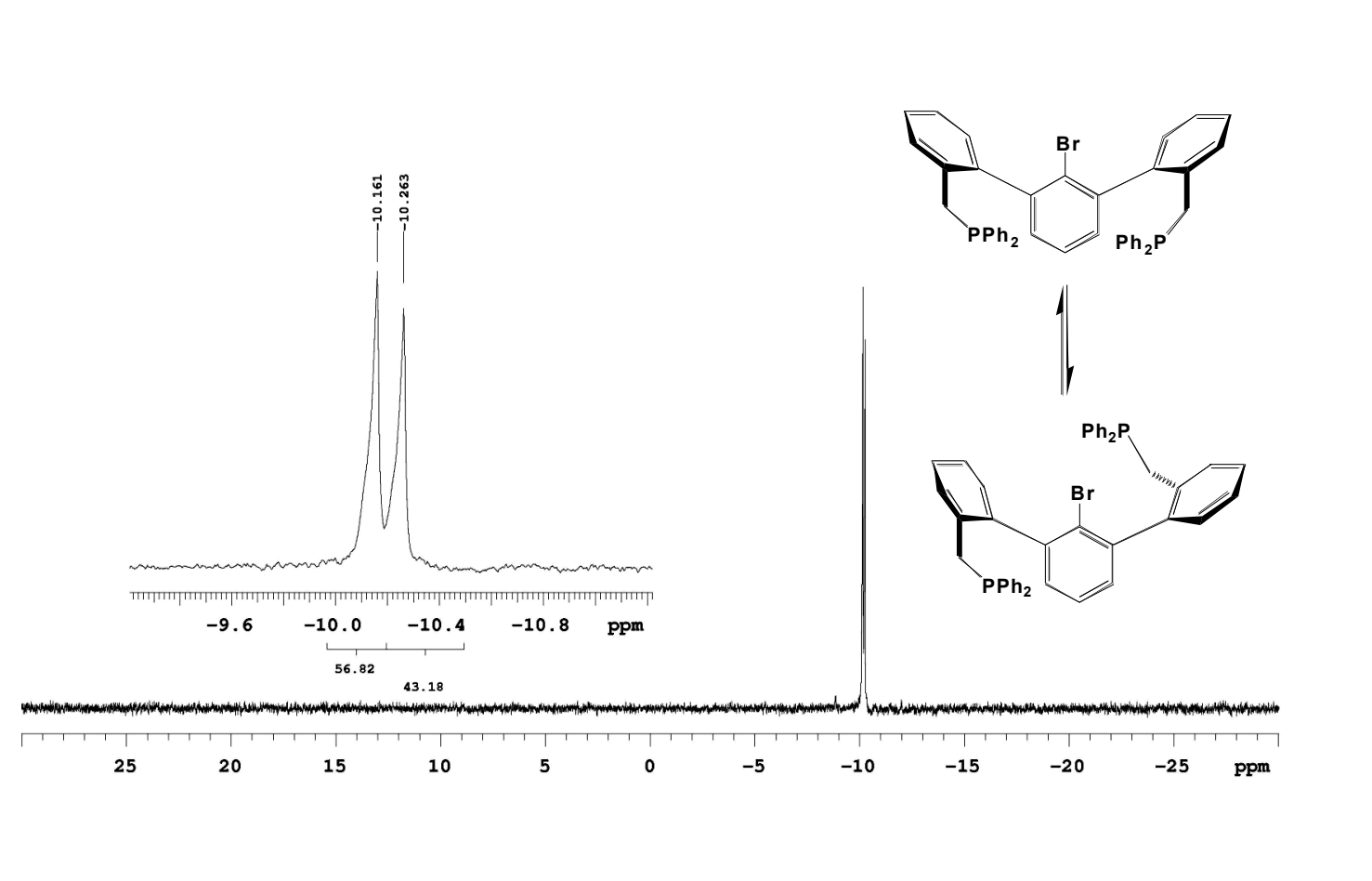

Figure S1. Two isomers (syn and anti) for diphosphine 1a as observed by ${ }^{31} \mathrm{P}$ NMR spectroscopy (161.2 MHz, $\left.\mathrm{C}_{6} \mathrm{D}_{6}\right)$. 

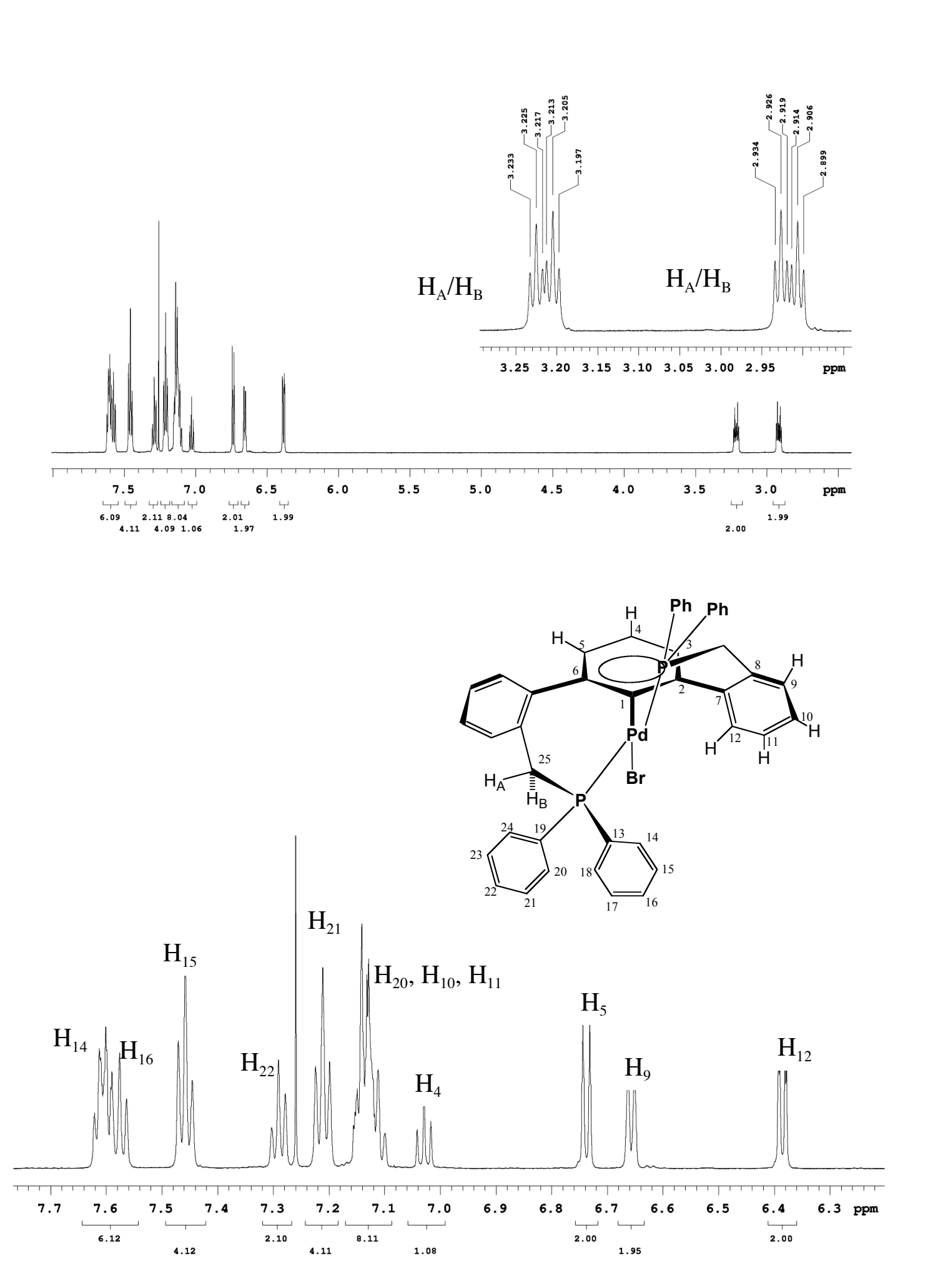

Figure S2. ${ }^{1} \mathrm{H}$ NMR spectra of $\mathbf{3 a}\left(\mathrm{CDCl}_{3}, 600 \mathrm{MHz}\right)$ 


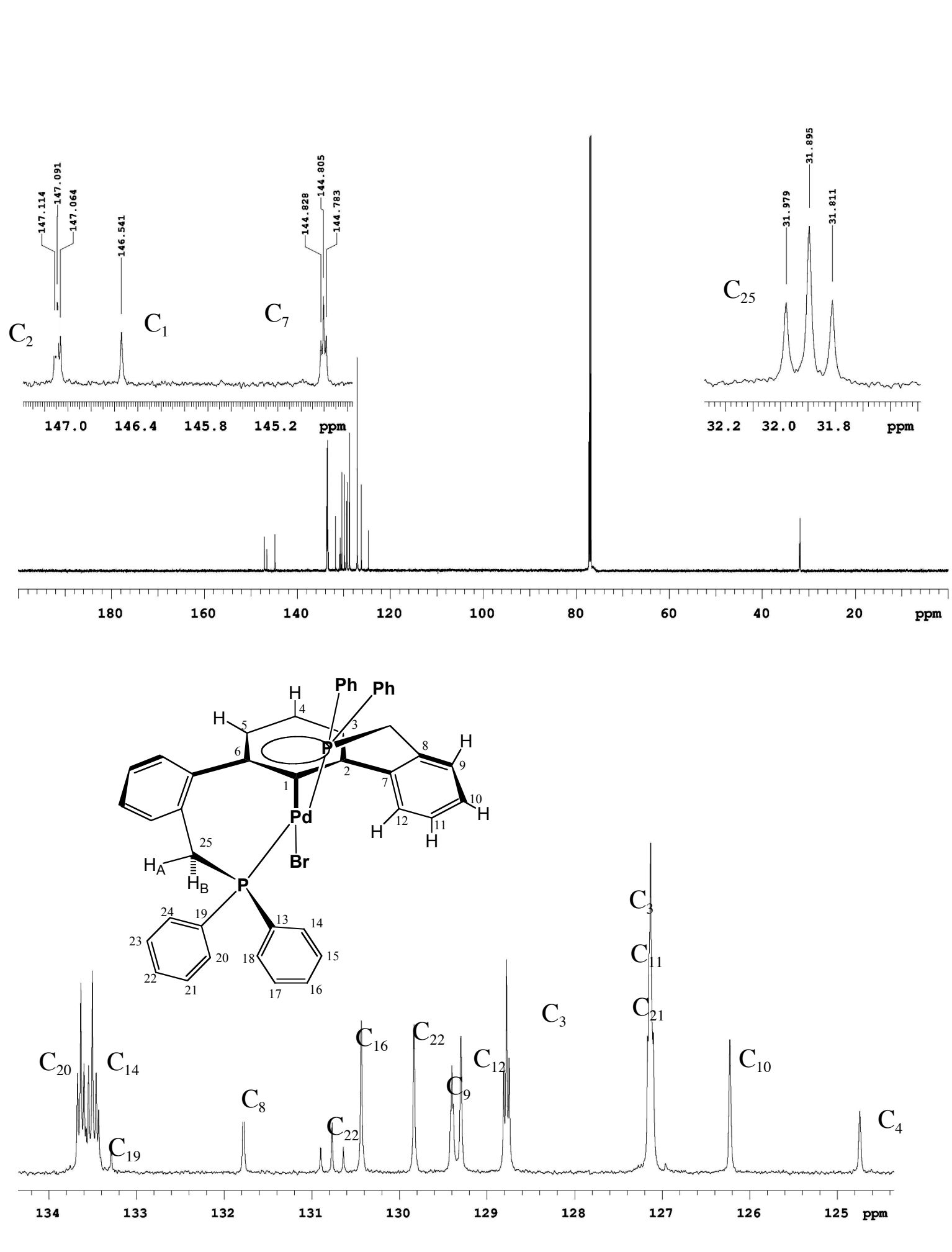

Figure S3. ${ }^{13} \mathrm{C}$ NMR spectra of $\mathbf{3 a}\left(\mathrm{CDCl}_{3}, 150 \mathrm{MHz}\right)$ 

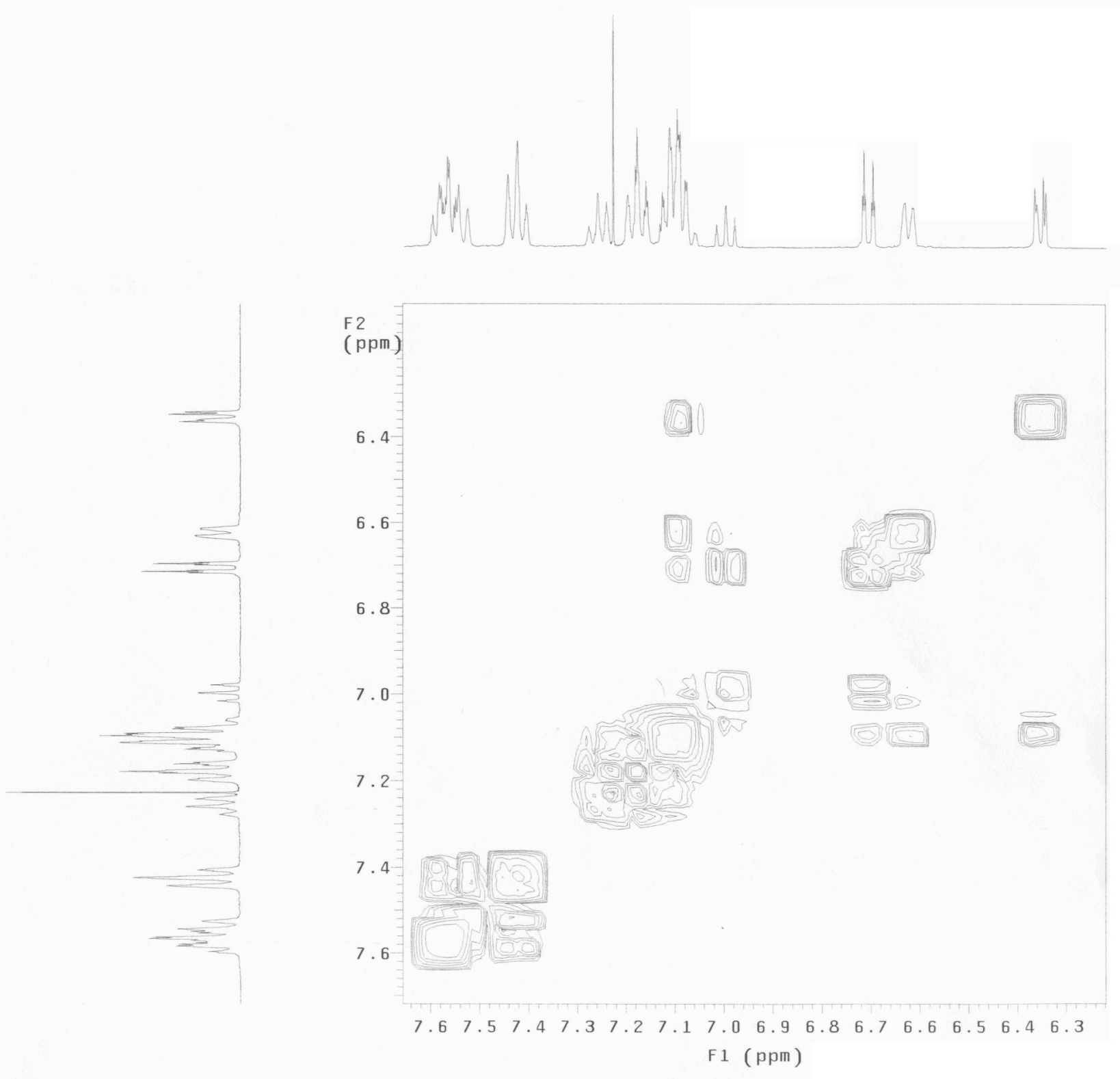

Figure S4. $600 \mathrm{MHz}{ }^{1} \mathrm{H}$ COSY spectrum of $\mathbf{3 a}\left(\mathrm{CDCl}_{3}\right)$. 


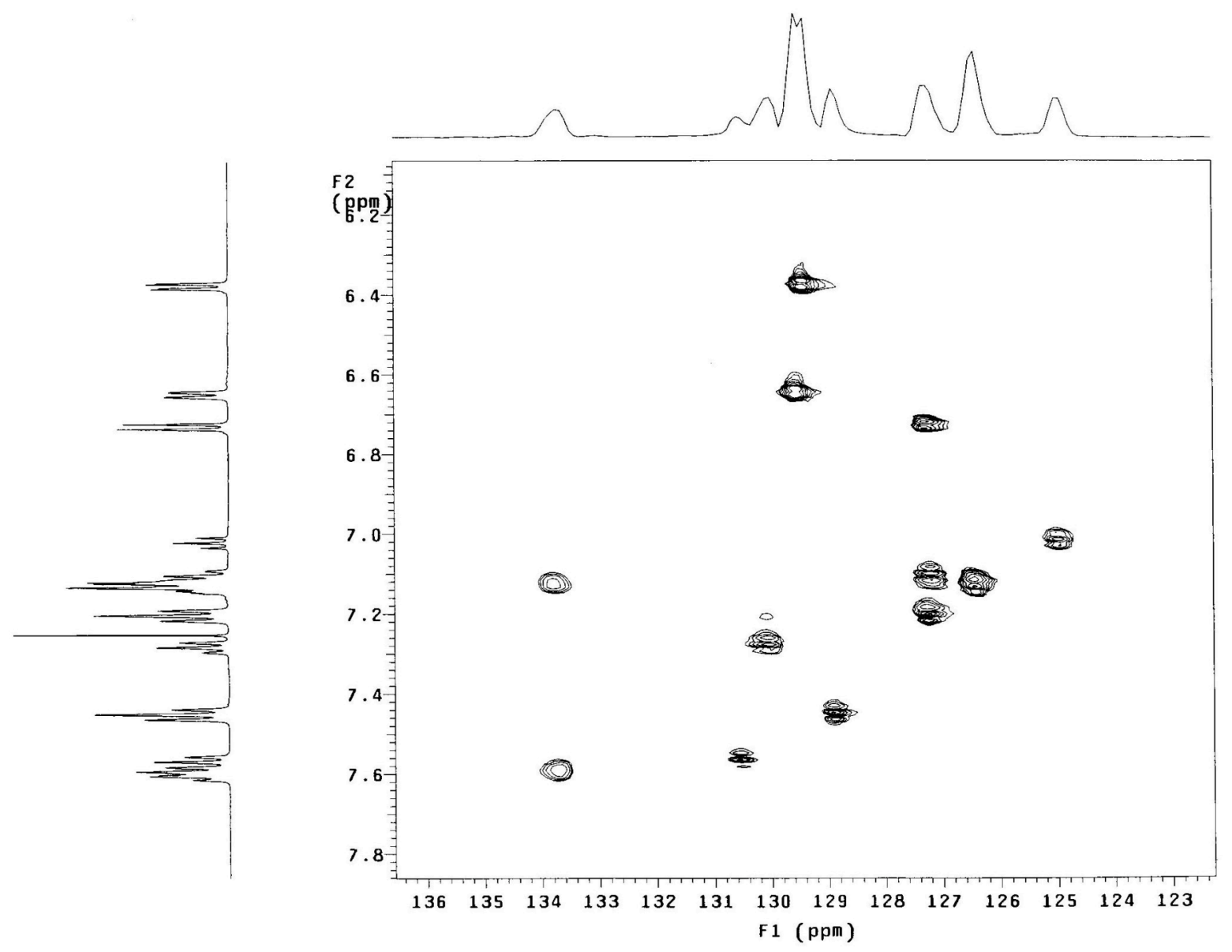

Figure S5. $600 \mathrm{MHz}{ }^{1} \mathrm{H}$-detected ${ }^{1} \mathrm{H}^{13} \mathrm{C}$ correlation (HMQC) spectrum of 3a. 


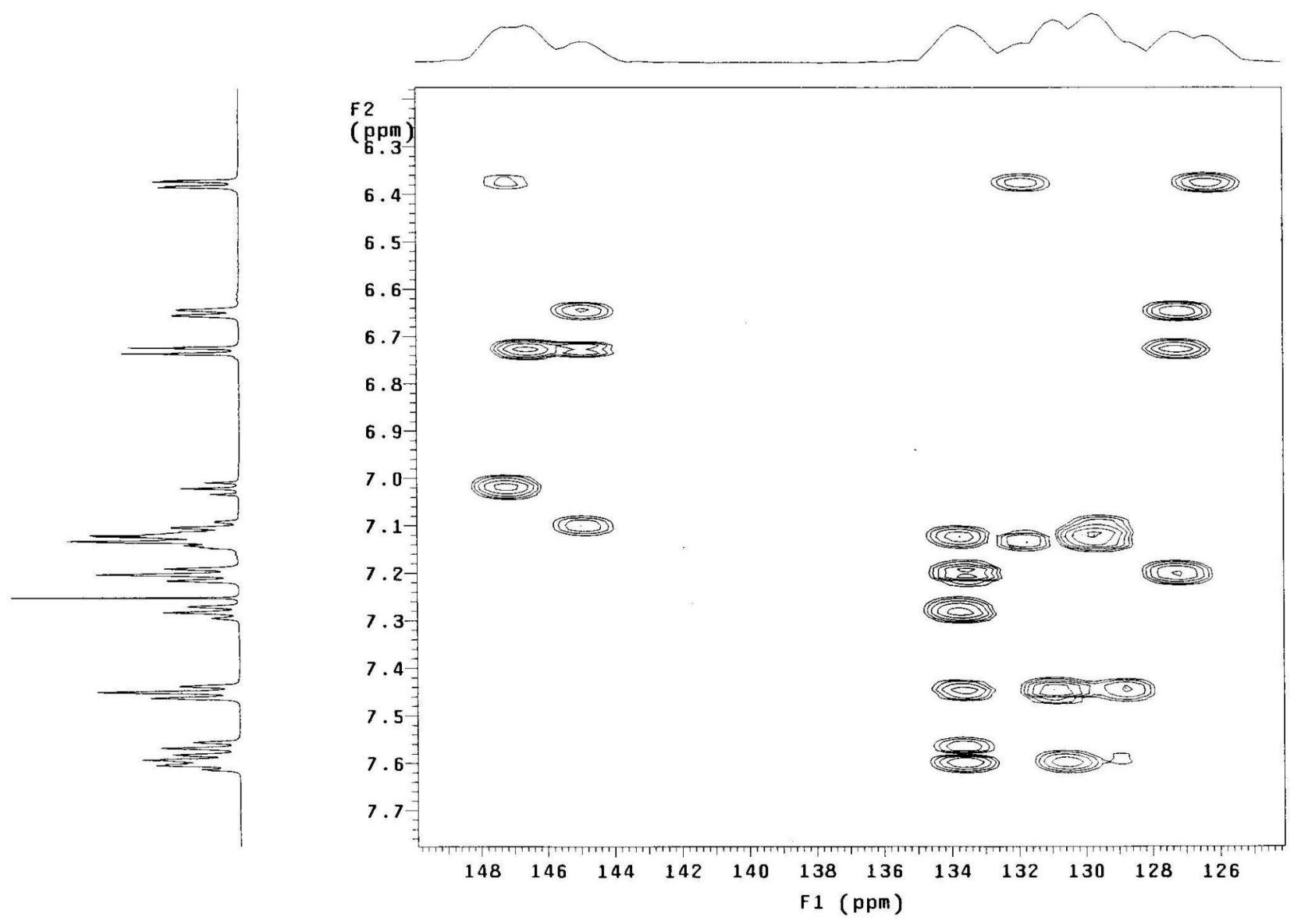

Figure S6. $600 \mathrm{MHz}{ }^{1} \mathrm{H}$-detected ${ }^{1} \mathrm{H}-{ }^{13} \mathrm{C}$ correlation (HMBC) spectrum of $\mathbf{3 a}$. 


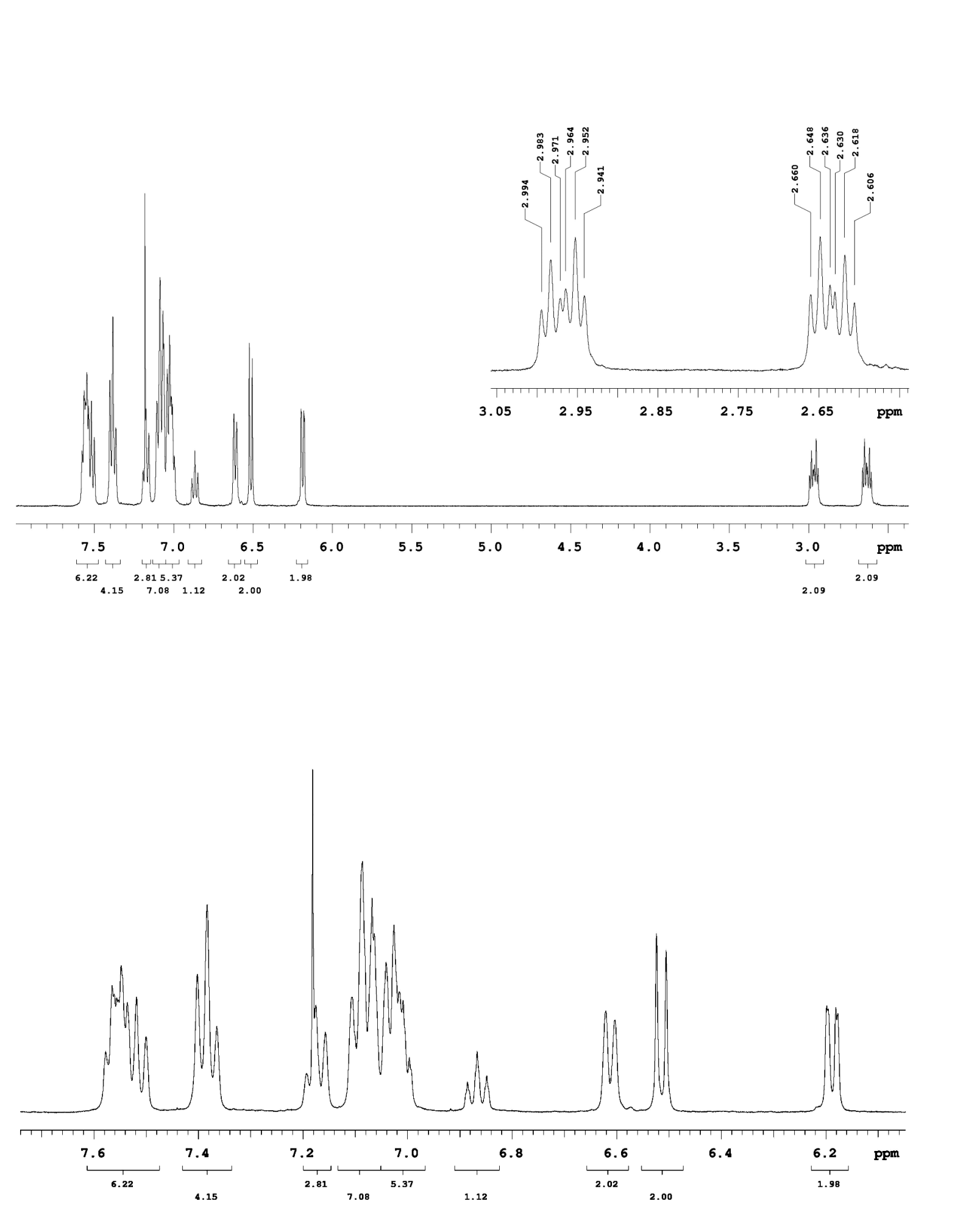

Figure S7. ${ }^{1} \mathrm{H}$ NMR spectra of $\mathbf{4 a}\left(\mathrm{CDCl}_{3}, 600 \mathrm{MHz}\right)$ 


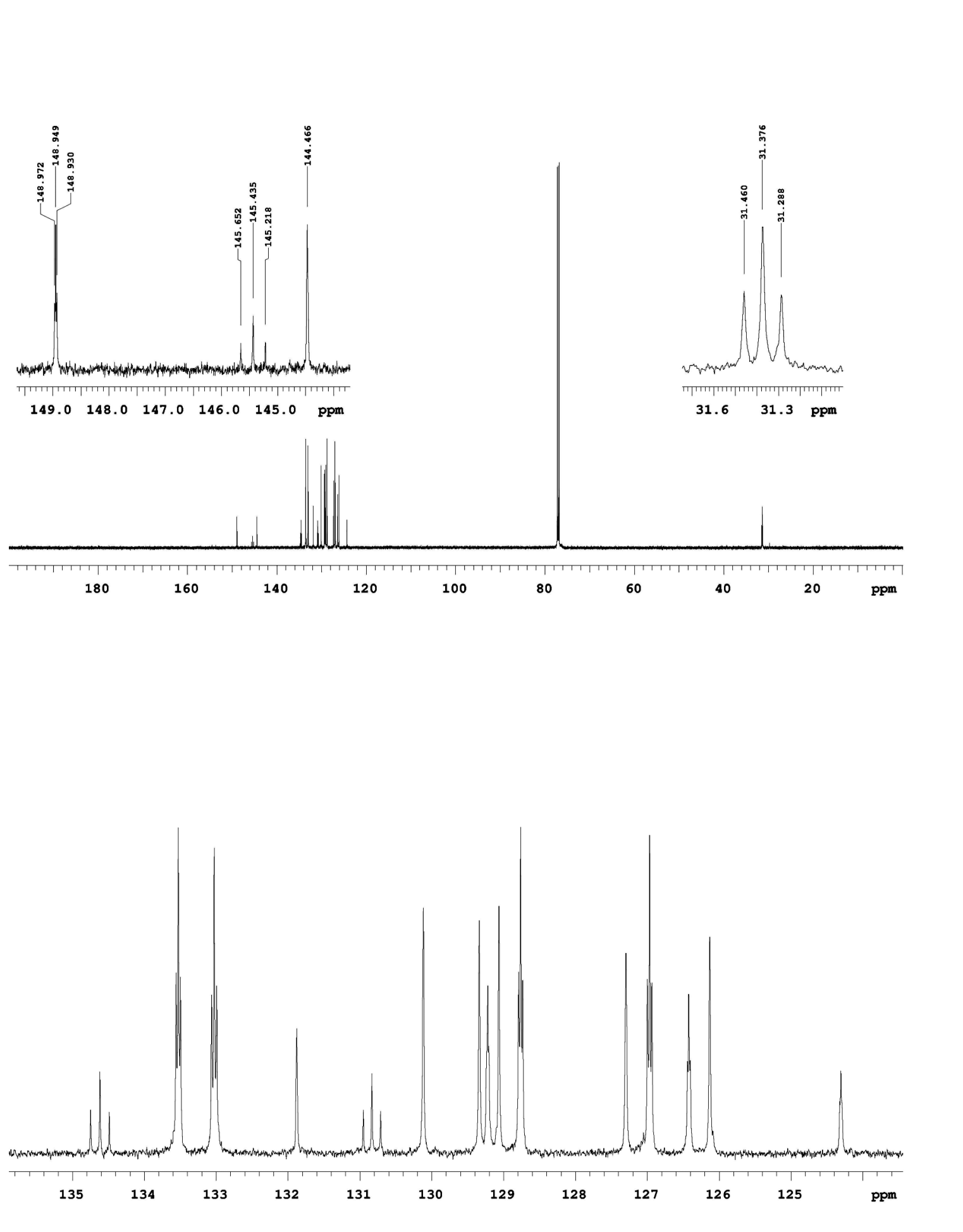

Figure S8. ${ }^{13} \mathrm{C}$ NMR spectra of $\mathbf{4 a}\left(\mathrm{CDCl}_{3}, 150 \mathrm{MHz}\right)$ 

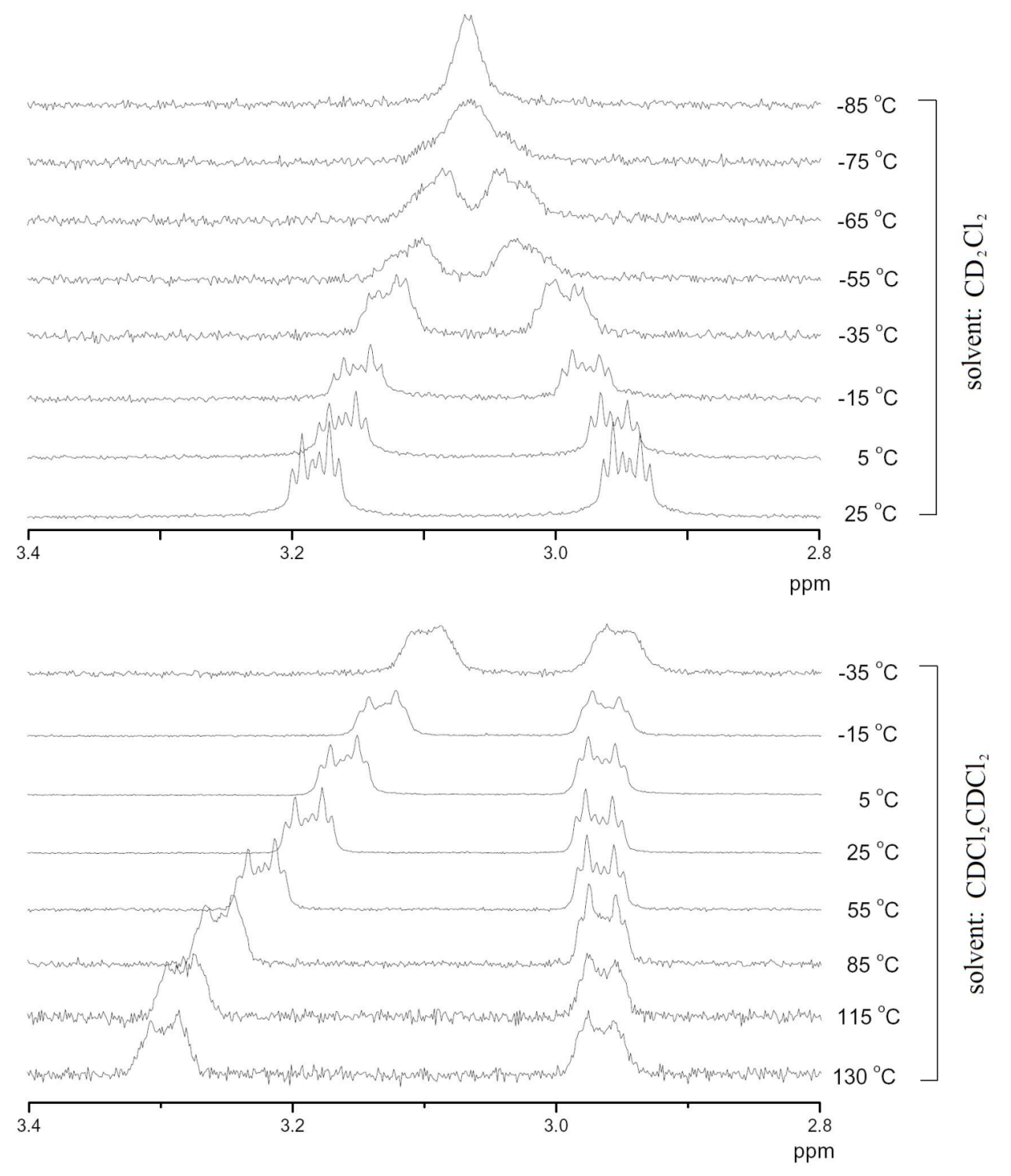

Figure S9. Variable temperature ${ }^{1} \mathrm{H} \mathrm{NMR}(600 \mathrm{MHz})$ of $3 \mathbf{a}$ in $\mathrm{CD}_{2} \mathrm{Cl}_{2}$ (top) and in $\mathrm{CDCl}_{2} \mathrm{CDCl}_{2}$ (bottom). 


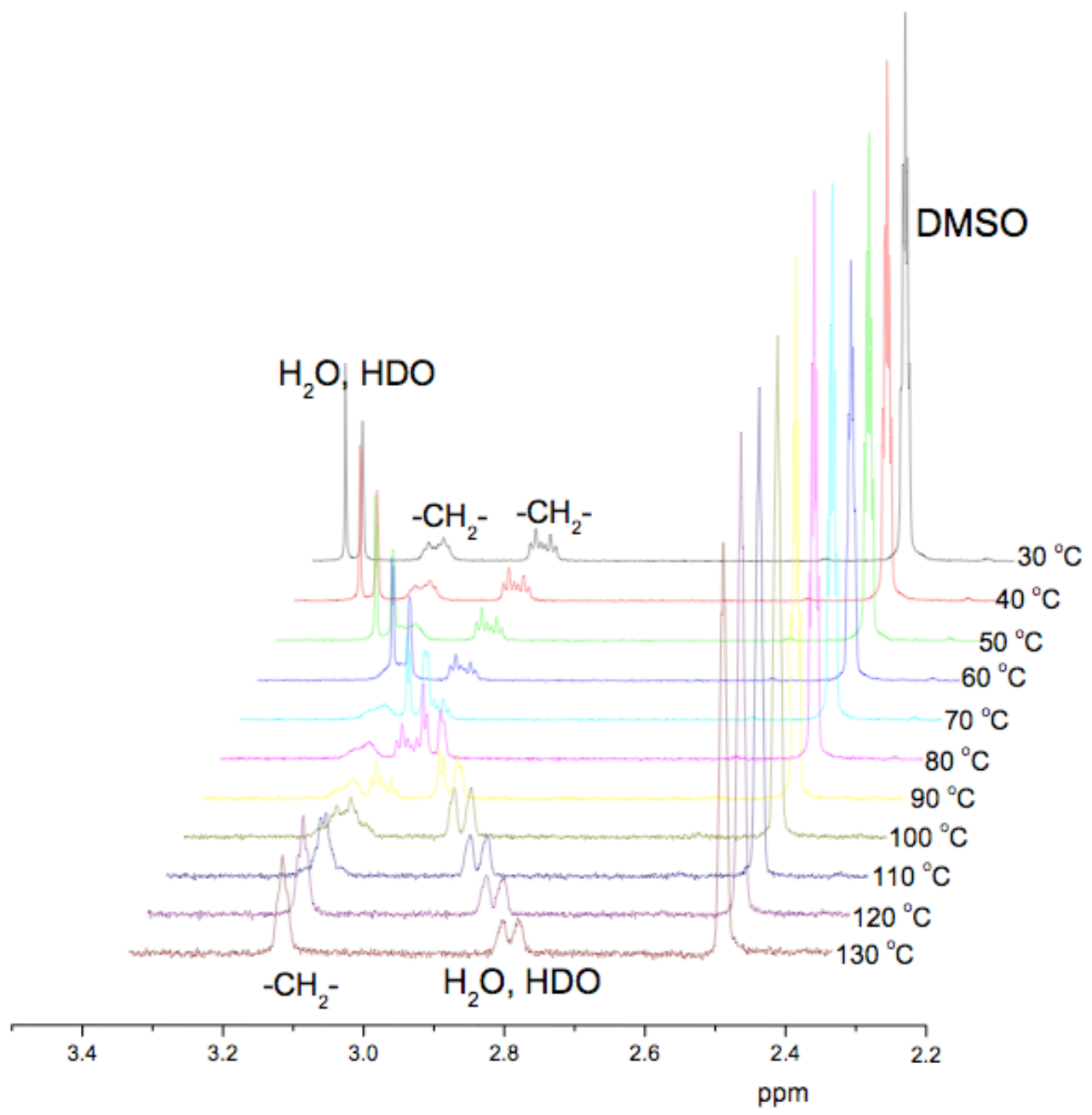

Figure S10. Variable temperature ${ }^{1} \mathrm{H}$ NMR (600 MHz, dmso-d6) of 3a for methylene region. 


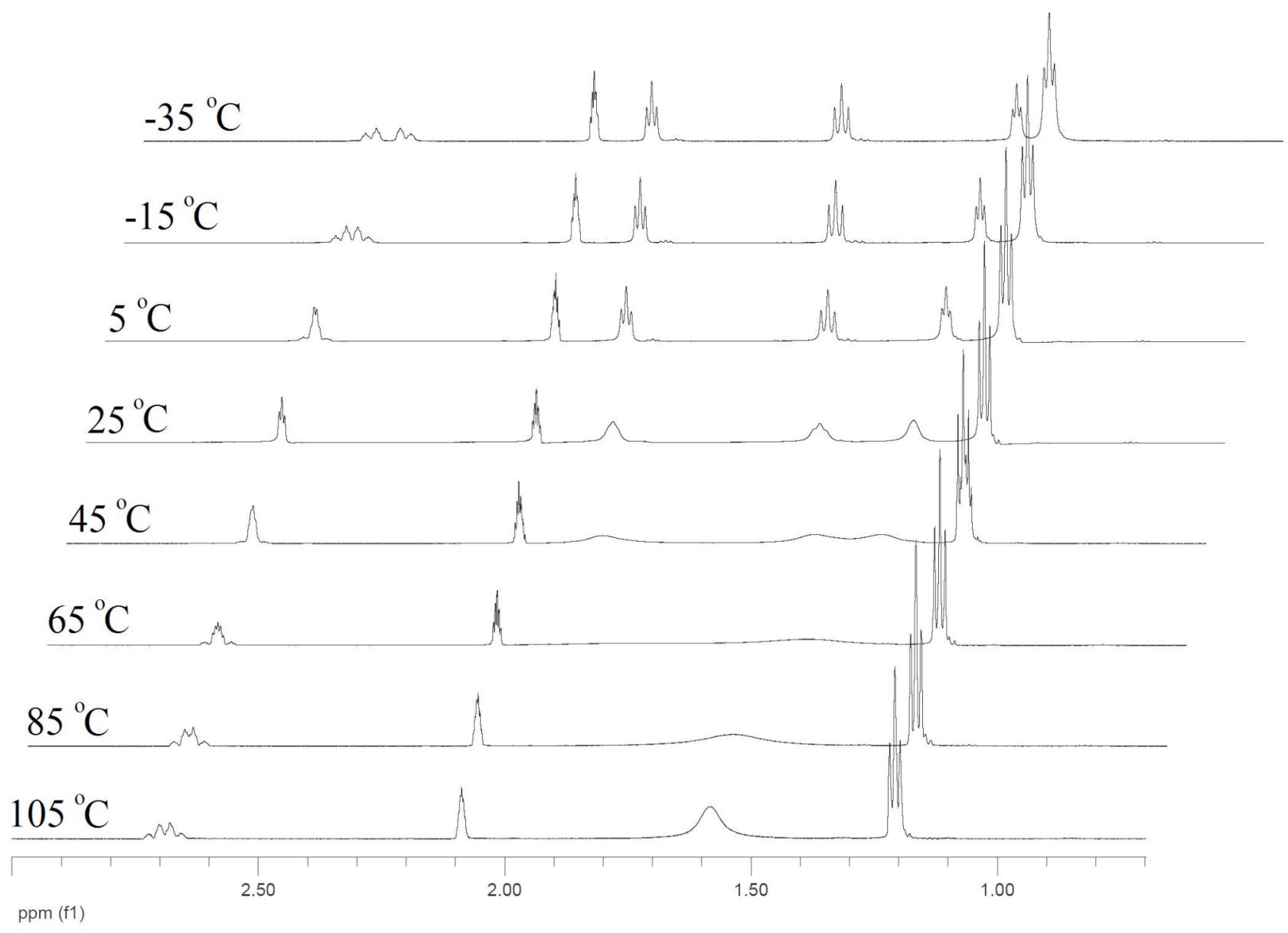

Figure S11. Variable temperature ${ }^{1} \mathrm{H}$ NMR $(600 \mathrm{MHz}$, toluene) of 3c for methylene region and tertbutyl region. 


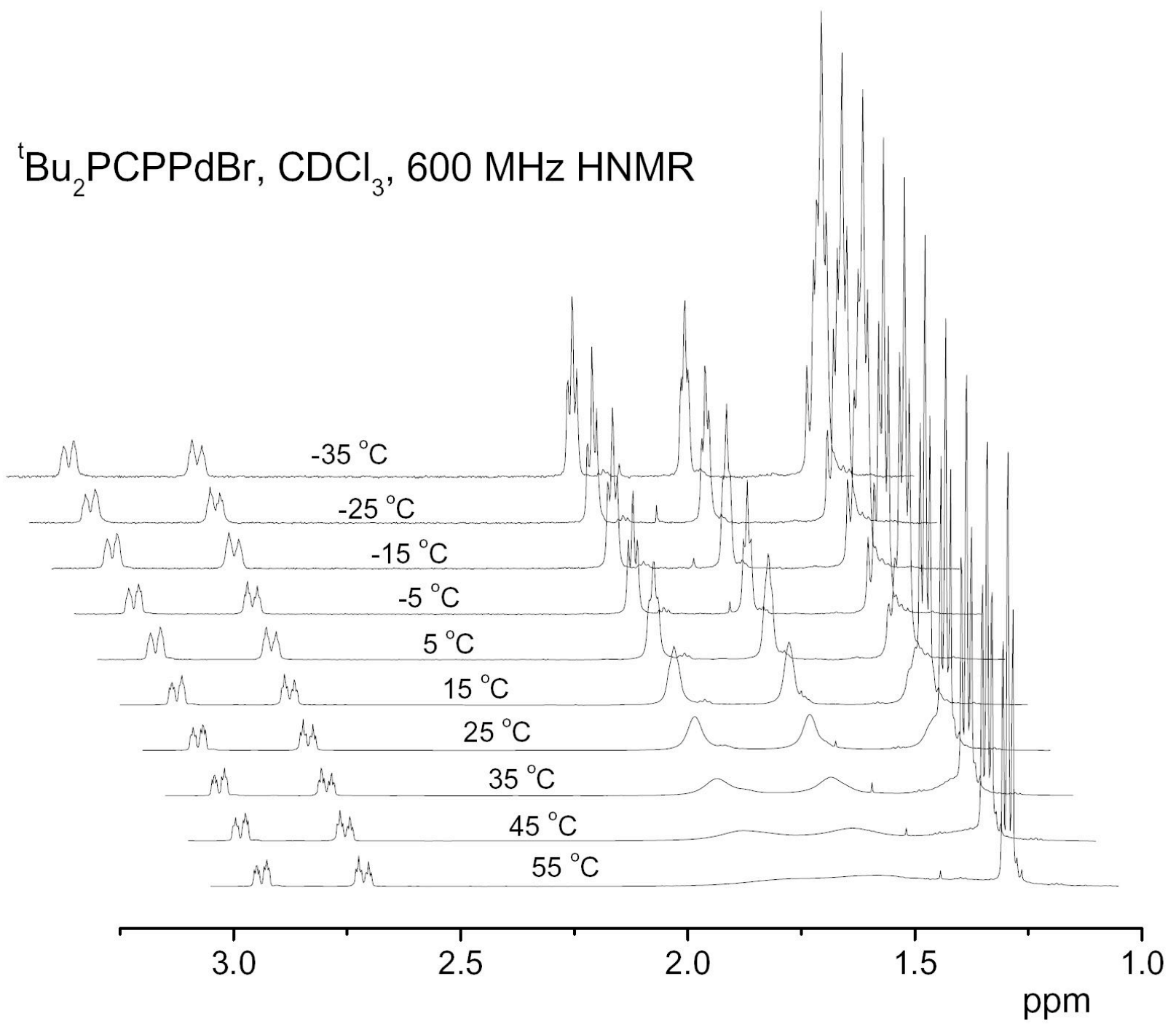

Figure S12. Variable temperature ${ }^{1} \mathrm{H} \mathrm{NMR}\left(600 \mathrm{MHz}, \mathrm{CDCl}_{3}\right)$ of $\mathbf{3 c}$ for methylene region and tertbutyl region. 